\title{
Age-dependent endocrine disorders involved in the pathogenesis of refractory acne in women
}

\author{
SIMONA IANOŞI ${ }^{1 *}$, GABRIEL IANOŞI $^{2^{*}}$, DANIELA NEAGOE ${ }^{3 *}$, OANA IONESCU $^{4 *}$, OVIDIU ZLATIAN $^{5 *}$, \\ ANCA OANA DOCEA ${ }^{6 *}$, CORIN BADIU $^{7 *}$, MARIA SIFAKI $^{8 *}$, DIMITRIS TSOUKALAS $^{8,9^{*}}$, \\ ARISTIDIS M. TSATSAKIS ${ }^{8 *}$, DEMETRIOS A. SPANDIDOS ${ }^{10^{*}}$ and DANIELA CĂLINA ${ }^{4 *}$
}

\begin{abstract}
Departments of ${ }^{1}$ Dermatology, ${ }^{2}$ Surgery, ${ }^{3}$ Internal Medicine, ${ }^{4}$ Clinical Pharmacy, ${ }^{5}$ Microbiology and ${ }^{6}$ Toxicology, University of Medicine and Pharmacy of Craiova, 200349 Craiova; ${ }^{7}$ Department of Endocrinology, Carol Davila University of Medicine and Pharmacy, 020021 Bucharest, Romania; ${ }^{8}$ Laboratory of Toxicology, Medical School, University of Crete, Heraklion 71003, Crete; ${ }^{9}$ Metabolomic Clinic, Kolonaki 10674, Athens; ${ }^{10}$ Laboratory of Clinical Virology, Medical School, University of Crete, Heraklion 71003, Crete, Greece
\end{abstract}

Received September 29, 2016; Accepted October 27, 2016

DOI: $10.3892 / \mathrm{mmr} .2016 .5924$

\begin{abstract}
Acne is a disorder of the pilosebaceous unit, common among adolescents, which may be extended to adulthood. The aim of this study was to assess the prevalence of hormonal disorders in women with acne resistance to conventional therapy. We included 72 women aged between 15 and 36 years (divided in two age groups) who presented to our clinic between May and October 2014, suffering from moderate and severe forms of papulopustular and nodulocystic acne. The subjects were non-responsive to classic dermatological treatment or had clinical manifestation of hyperandrogenism. Based on age, we divided the women into two groups, group I with 40 patients aged 15-22 years and group II with 32 patients aged 23-36 years. Using ELISA, a hormonal profile was performed for each patient in days 1-3 of the menstrual cycle including, total testosterone, dehydroepiandrosterone sulfate (DHEA-S), follicle-stimulating hormone (FSH), luteinizing hormone ( $\mathrm{LH}$ ), estradiol, prolactin, and plasma cortisol. For statistical analysis we used Stata 13 software. We compared the hormonal profile of the two groups and identified significant differences for: testosterone levels (mean value, $0.64 \pm 0.35$ vs. $0.97 \pm 0.50 \mathrm{ng} / \mathrm{ml}$; $\mathrm{p}<0.0001$ ), DHEA-S levels (mean value, $0.85 \pm 0.27$ vs. 1.05
\end{abstract}

Correspondence to: Dr Anca Oana Docea, Department of Toxicology, University of Medicine and Pharmacy of Craiova, 2 Petru Rareş Street, 200349 Craiova, Romania

E-mail: ancadocea@gmail.com

Dr Gabriel Ianoşi, Department of Surgery, University of Medicine and Pharmacy of Craiova, 2 Petru Rareş Street, 200349 Craiova, Romania

E-mail: gianosi@hotmail.com

*Contributed equally

Key words: acne, hormonal profile, women $\pm 0.33 \mathrm{mg} / 24 \mathrm{~h} ; \mathrm{p}=0.001$ ), prolactin levels (mean value, 281 . $85 \pm 91.113$ vs. $353.969 \pm 102.841 \mathrm{mIU} / \mathrm{ml} ; \mathrm{p}=0.002)$ and $\mathrm{LH}$ levels $(14.8 \pm 6.7$ vs. $20.1 \pm 8.2 \mathrm{mIU} / \mathrm{ml}$; $\mathrm{p}=0.002)$ were higher in group II. No statistically significant differences were found for estradiol $(\mathrm{p}=0.588)$ and cortisol $(\mathrm{p}=0.182)$ levels. In conclusion, refractory acne can be the first sign of systemic illness including polycystic ovary syndrome. Thus, for a correct therapeutic approach it is necessary to interpret the clinical and biochemical elements in correlation with the medical history.

\section{Introduction}

Acne is a common skin condition occurring at the transition between puberty and adult age, which is quasi-physiological and usually self-limited. Currently, there are different clinical forms of acne. Acne vulgaris is the most common form, but there are also some difficulties in diagnosing forms such as fulminans acne (1), whose diagnosis often requires skin biopsy. It is crucial to distinguish the border between the acne specific to age and that of pathological condition because, under certain circumstances, acne can be a cutaneous expression of hormonal disorders (2).

Women have three major sources of androgens (3): i) the ovaries, stimulated by the pituitary hormones follicle-stimulating hormone (FSH) and luteinizing hormone ( $\mathrm{LH})$, which produce small quantities of androgens [dehydroepiandrosterone sulfate (DHEA-S) and testosterone] that can be released per se in the circulation or converted into estrogen by the enzyme aromatase, which is present in the ovarian follicle cells. At this level, disorders of androgen excess are represented by functional ovarian hyperandrogenism, whereas androgen-secreting tumors occur rarely. ii) The adrenal gland produces DHEA-S which can be metabolized in more potent androgens such as androstenedione and testosterone; and iii) the skin, which has all the enzymes required for converting the weak androgens into strong androgens such as testosterone and in the synthesis of androgens. 
In sebaceous glands, the increased activity of these enzymes sustains the major role of androgens in inducing skin lesions. Thus persistent acne can be explained in adult women with high levels of testosterone and DHEA-S, which are practically the most important hormones for the diagnosis of endocrine acne $(2,3)$.

According to the Global Acne Grading System (GAGS), each type of acneiform lesion has a gravity score: no lesions, 0 ; comedones, 1; papules, 2; pustules, 3; and nodules, 4 . The local score was calculated using the formula: Factor $x$ grade $0-4$ Depending on the location of acne, the factor had the following values: forehead, 2; right cheek, 2; left cheek, 2; chin, 1; thorax and upper torso, 1 . The sum of the local scores was the global score which settled acne severity. A global score of 1-18 signified mild acne; 19-30, moderate acne; 31-38, severe acne; and a global score $>39$, very severe acne (4).

The persistence of acne in adulthood or its late onset (in women $>25$ years) suggests an endocrine cause due to hyperandrogenism (5). Although the most common cause of hyperandrogenism is represented by PCOS, the differential diagnoses with Cushing's syndrome, ovarian or adrenal androgen-secreting tumors, acromegaly or with non-endocrine disorders, Apert syndrome, Behçet's syndrome and SAHA syndrome (seborrhoea, acne, hirsutism and alopecia) are of importance (6).

The diagnosis of PCOS should be suspected in the presence of hyperandrogenism and the following clinical manifestations: severe acne that reoccurs after isotretinoin therapy associated with hirsutism, oligomenorrhea or amenorrhea (defined as the presence of $<8$ menstrual cycles per year), androgenic alopecia, seborrhea and acanthosis nigricans on the backhead, digits, inguinal or periocular - an insulin resistance marker. Those clinical signs must also be correlated with laboratory tests for hyperandrogenism and with transvaginal and pelvic ultrasound (7).

The aim of the present study was to assess the prevalence of hormonal profile disturbances according to age in women with papulopustular and nodulocystic acne resistant to conventional therapy (retinoid therapy, topical benzoyl peroxide and azelaic acid, local and/or systemic antibiotherapy or isotretinoin).

\section{Materials and methods}

Patient data. This observational cross-sectional study included 72 patients, aged 15-36 years, who were tested between May and October 2014 in the Department of Dermatology, Emergency Regional Hospital (Craiova, Romania). The patients suffered from moderate and severe forms of papulopustular and nodulocystic acne and were unresponsive to classical dermatological treatment or had clinical manifestation of hyperandrogenism. The patients were divided into two age groups: the first one (I) included 40 patients, aged 15-22 years, and the second one (II) included 32 patients, aged 23-36 years.

Informed consent was obtained from each patient $>18$ years of age and parental informed consent for those $<18$ years was obtained. The study was conducted in accordance with the World Medical Association Declaration of Helsinki and approved by the Institutional Ethics Committee of the Emergency Regional Hospital.
Inclusion criteria for the sudy were: acne resistant to conventional dermatological therapy (retinoid therapy, topical benzoyl peroxide and azelaic acid, local and/or systemic antibiotherapy or isotretinoin); acne accompanied by a hyperandrogenic status: hirsutism, intense facial seborrhea, irregular menses, androgenic alopecia, voice changes; refractive acne with polycystic ovaries evidenced on endovaginal ultrasound; sudden onset of acne in women aged $>23$ years, unresponsiveness to local and/or systemic antibiotherapy or isotretinoin therapy, and a body mass index (BMI) of $>29 \mathrm{~kg} / \mathrm{m}^{2}$.

Exclusion criteria for the study were: pregnant women, nursing mothers, cosmetic acne, rosacea, seborrheic dermatitis, patients with functional hyperprolactinemia due to estrogen therapy (oral contraceptives in the last 3 months), patients under treatment with drugs that cause acne (progesterone, glucocorticoids, lithium, selective reuptake serotonin inhibitors, isoniazid, phenytoin as well as vitamins B2, B6 and B12) and other medication including: dopamine antagonists (phenothiazines, haloperidol, metoclopramide, reserpine, methyldopa), cimetidine, verapamil, and monoamine oxidase inhibitors.

Endocrine evaluation. A hormonal profile was performed on each patient, including $\mathrm{FSH}, \mathrm{LH}$, estradiol, prolactin, total testosterone, DHEA-S and plasma cortisol using ELISA (ELx808IU device; BioTek, Winooski, VT, USA).

Testosterone normal levels for women were considered as $\leq 0.9 \mathrm{ng} / \mathrm{ml}$. Excluding adrenal disorders, an increased serum level of testosterone indicated an ovarian source. Moderate increases of $1.5 \mathrm{ng} / \mathrm{ml}$ were suggestive for PCOS, whereas values $>20 \mathrm{ng} / \mathrm{ml}$ strongly suggested androgen-secreting tumors. DHEA-S (normal levels $\leq 1 \mathrm{mg} / 24 \mathrm{~h}$ ) and serum testosterone were used as a screening test for acne associated with hirsutism. Approximately $84 \%$ of women with hirsutism have high levels of androgens. High levels of DHEA-S are typical for PCOS (9).

Normal levels of FSH (follicular phase) were settled as $\leq 15 \mathrm{mIU} / \mathrm{ml}$, while $\mathrm{LH}$ was $\leq 25 \mathrm{mIU} / \mathrm{ml}$. An LH/FSH ratio $>2$ was considered abnormal and was associated with PCOS albeit it is not characteristic of PCOS. Additionally, women with PCOS with a normal LH/FSH ratio were reported to be overweight and have insulin resistance (10).

The particular role of androgen excess in the etiology of refractive acne is well known, while the role of estrogen is incompletely elucidated. Estrogens increase sex hormonebinding globulin (SHBG) acting through liver (11). SHBG has a high binding affinity for testosterone, and an outstanding estrogen affinity. Since testosterone and dihydrotestosterone are the primary androgens involved in the etiology of refractory acne, decreased levels of SHBG lead to poor response to treatment (12).

On the other hand, the role of estrogens in modulating the sebum production is little understood. To reduce the sebum production, an increased quantity of estrogens is necessary, greater than the one necessary for the inhibition of ovulation, administered for a long time. In the present study, we evaluated estradiol levels during the follicular phase of the menstrual cycle (normal range, 46-607 pmol/1).

Prolactin levels (normal range, 132-498 mIU/l) were also measured considering a number of studies that reported high 


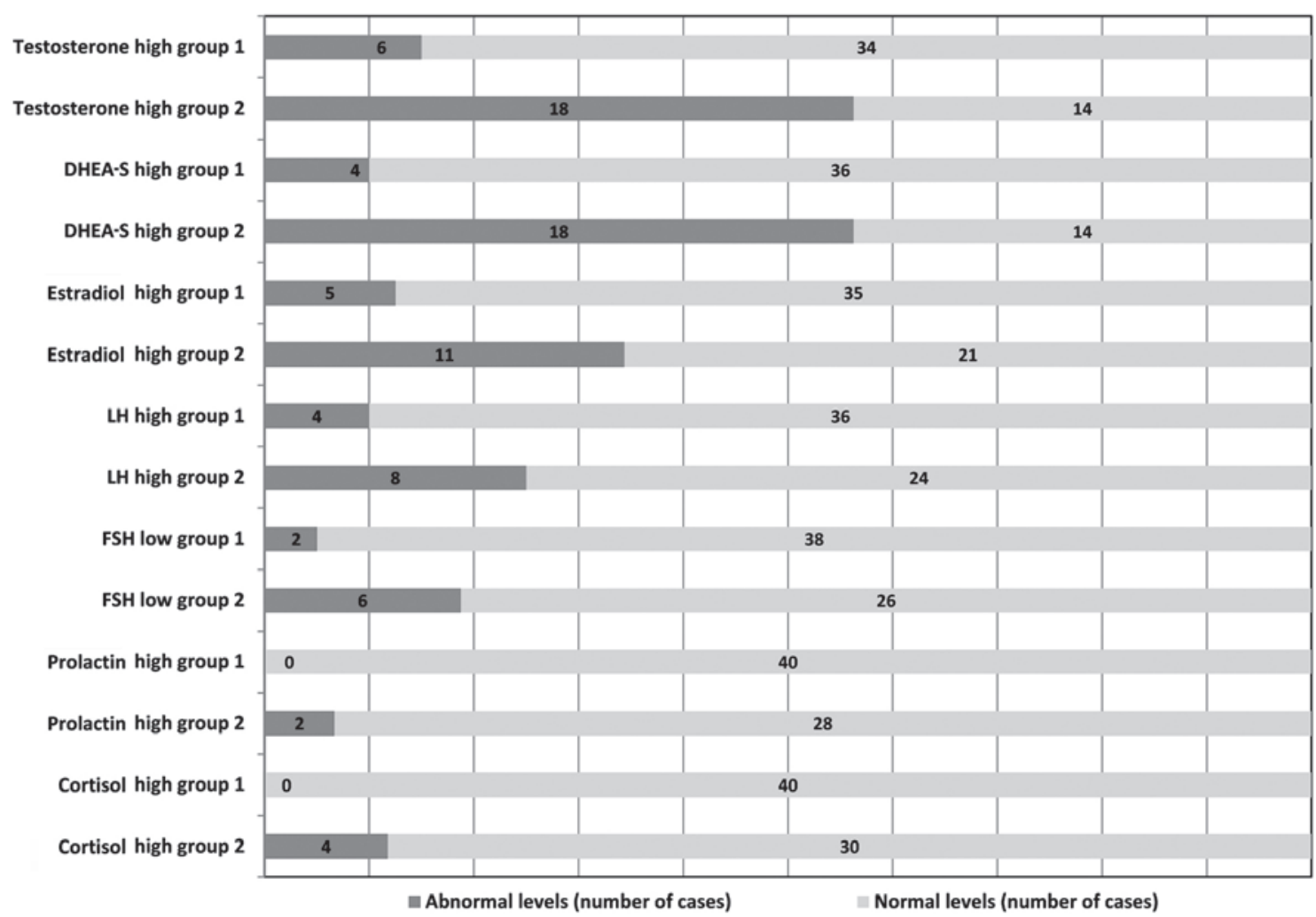

Figure 1. Hormonal disturbances in patients from group I (15-22 years) comparative with group II (23-36 years). We accounted testosterone high $>0.9 \mathrm{ng} / \mathrm{ml}$, DHEA-S high $>1 \mathrm{mg} / 24 \mathrm{~h}$, estradiol high $>607 \mathrm{pmol} / \mathrm{l}$ (in follicular phase), LH high $>25 \mathrm{mIU} / \mathrm{ml}$, FSH low $<15 \mathrm{mIU} / \mathrm{ml}$, prolactin high $>498 \mathrm{mIU} / \mathrm{ml}$ and cortisol high $>230 \mathrm{ng} / \mathrm{ml}$. DHEA-S, dehydroepiandrosterone-sulfate; LH, luteinizing hormone; FSH, follicle-stimulating hormone.

levels of prolactin in women with PCOS and refractive acne (13). PCOS occurs as a consequence of multiple factors including the abnormal release of gonadotropins and ovarian dysfunction. Alteration in dopamine turnover can also perturb the release of gonadotropins, being a common cause for both PCOS and hyperprolactinemia (14). However, hyperprolactinemia is most often transitory, as a very small percentage of patients with PCOS and hyperprolactinemia have high levels of prolactin. Other studies reported no relationship between PCOS and hyperprolactinemia, while high levels of estrogens stimulate moderate prolactin secretion (14).

Serum cortisol measurement was performed at 8:00 a.m. and normal levels were settled at 5-23 $\mu \mathrm{g} / \mathrm{dl}$. A moderate increase was indicated when double levels occurred in PCOS, and high levels were most frequently associated with Cushing's syndrome (15). In these cases a differential diagnosis with Cushing's and pseudo-Cushing's syndromes using a dexamethazone suppression test was performed. In addition, hypercortisolism was predisposed to excessive cutaneous sebum secretion and through immunosuppression allowed the bacteria Propionibacterium acnesto to multiply, causing acne. It is well known that the plasmatic cortisol is increased during stress, explaining the exacerbation of acne in stressful periods (16).

Statistical analysis. Data entry and statistical processing were performed using Stata 13 software (StataCorp LP, College Station, TX, USA). The correlations between continuous variables were assessed using the Pearson's correlation coefficient $\mathrm{r}$ and were graphically represented through scatter $\mathrm{p}$ groups with superimposed linear fit curves. $\mathrm{P}<0.05$ was considered or all the statistical tests.

\section{Results}

Hormonal disturbances. In the first group of 40 patients aged 15-22 years we observed the following hormonal disturbances: increased total testosterone in 6 patients $(15 \%)$, increased DHEA-S in 4 patients $(10 \%)$ that presented oligomenorrhea associated with an intense seborrheic secretion, increased estradiol in 5 patients $(12.5 \%)$, decreased FSH in 2 patients (5\%) and increased LH in 4 patients (10\%) (Fig. 1). Serum cortisol and prolactin levels were within normal range.

In the second group of 32 patients aged 23-36 years, 6 patients had refractory acne (persistent acne), 10 patients had moderate acne (and sudden onset after age of 23) and 16 patients had refractory acne and at least one sign of hyperandrogenism (irregular menstrual cycles in 10 patients, intense seborrheic status associated with deepening of the voice, and androgenic alopecia or hirsutism in 12 patients). In this group, we found the following hormonal disturbances: increased total testosterone in 18 patients $(56.25 \%)$, increased DHEA-S in 18 patients $(56.25 \%)$. From the last 18 patients, 11 (61.11\%) had refractory acne associated with the clinical manifestation of hyperandrogenism and 7 of them $(38.8 \%)$ had refractory late-onset (after 23 years) acne associated without clinical manifestation of hyperandrogenism. Eight patients who experienced clinical hyperandrogenism showed normal values of total testosterone and DHEA-S, probably through an increase of the cutaneous androgen receptor number or through an 
Table I. Clinical and laboratory features of patients with refractory acne.

\begin{tabular}{lccr}
\hline & $\begin{array}{c}\text { Mean } \pm \text { SD } \\
\text { Parameter }\end{array}$ & $\begin{array}{c}\text { Mean } \pm \text { SD } \\
\text { (group I: } 40 \text { patients) }\end{array}$ & P-value \\
\hline Age $($ years) & $18.30 \pm 2.311$ & $31.25 \pm 4.683$ & $<0.001$ \\
BMI $\left(\mathrm{kg} / \mathrm{m}^{2}\right.$ ) & $20.321 \pm 6.782$ & $25.789 \pm 7.751$ & 0.021 \\
Testosterone $(\mathrm{ng} / \mathrm{ml})$ & $0.642 \pm 0.352$ & $0.971 \pm 0.505$ & $<0.001$ \\
DHEA-S $(\mathrm{mg} / 24 \mathrm{~h})$ & $0.854 \pm 0.279$ & $1.056 \pm 0.331$ & $<0.001$ \\
Estradiol $(\mathrm{pmol} / \mathrm{l})$ & $380.625 \pm 223.535$ & $410.531 \pm 237.838$ & 0.588 \\
FSH $(\mathrm{mIU} / \mathrm{ml})$ & $6.31 \pm 3.579$ & $4.545 \pm 3.566$ & 0.041 \\
LH $(\mathrm{mIU} / \mathrm{ml})$ & $14.875 \pm 6.711$ & $20.156 \pm 8.215$ & 0.004 \\
Prolactin $(\mathrm{mIU} / \mathrm{ml})$ & $281.85 \pm 91.113$ & $353.969 \pm 102.841$ & 0.002 \\
Serum cortisol $(\mu \mathrm{g} / \mathrm{dl})$ & $155.45 \pm 46.568$ & $137.625 \pm 61.901$ & 0.182 \\
\hline
\end{tabular}

BMI, body mass index; DHEA-S, dehydroepiandrosterone sulfate; FSH, follicle-stimulating hormone; LH, luteinizing hormone.
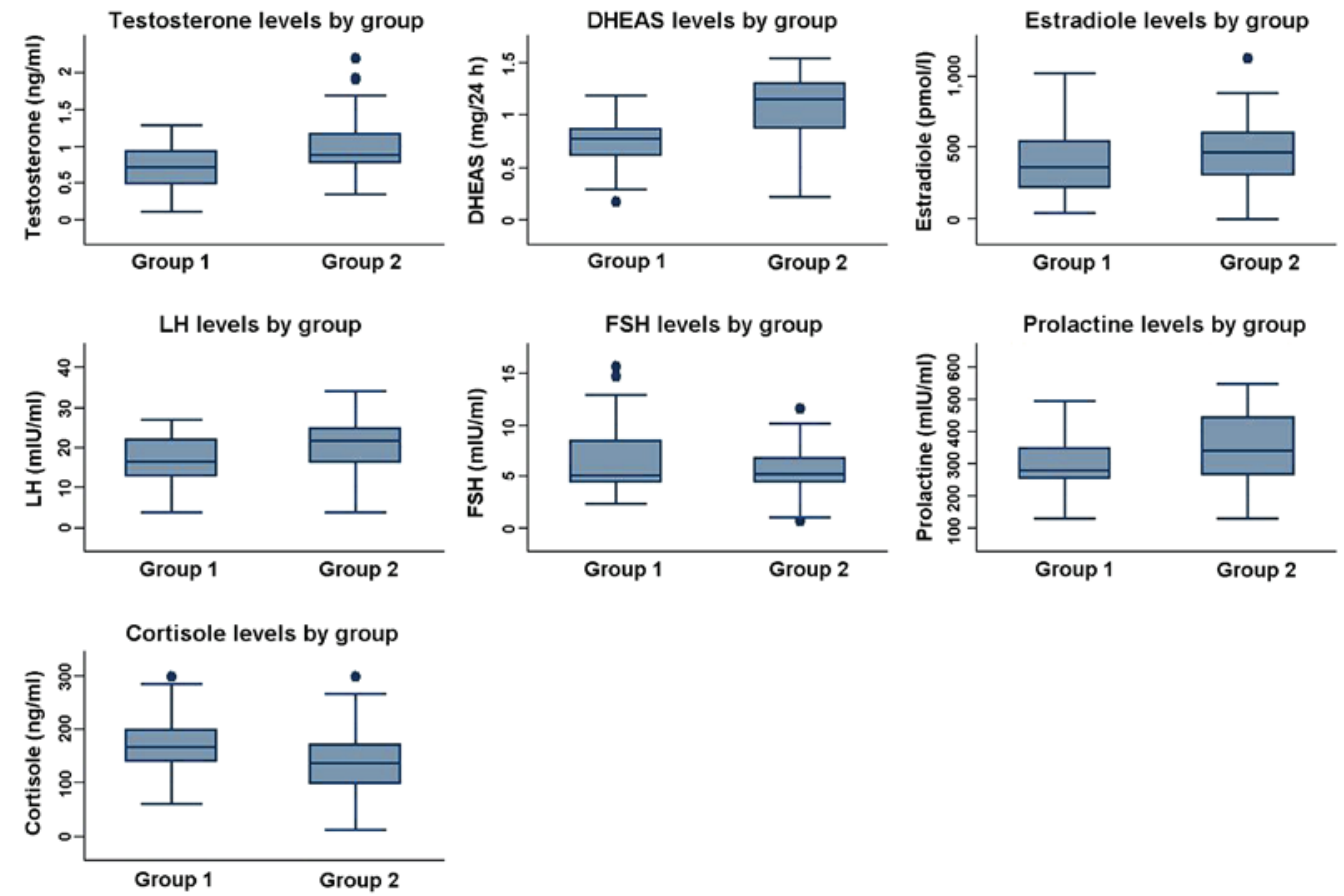

Figure 2. Distribution of the hormone levels in the two groups. Single dot, low outlier value; double dot, high outlier value.

increased receptor sensitivity. Estradiol levels were increased in 11 patients $(34.3 \%)$, LH levels were increased in 8 patients (25\%), hyperprolactinemia occurred in 2 patients $(6.25 \%)$ and hypercortisolism in 4 patients $(12.5 \%)$. FSH levels were decreased in 6 patients $(18.75 \%)$ (Fig. 1).

Clinical features of patients and hormone level distribution. Demographic and biochemical data analysis are shown in Table I and Fig. 2.

Testosterone levels were lower in group I $(0.642 \pm 0.352 \mathrm{ng} / \mathrm{ml})$ vs. group II $(0.972 \pm 0.505 \mathrm{ng} / \mathrm{ml})$, and the differences were highly significant $(\mathrm{p}<0.0001)$. DHEA-S was also decreased in group I $(0.854 \pm 0.279 \mathrm{mg} / 24 \mathrm{~h}) \mathrm{vs}$. group II $(1.056 \pm 0.331 \mathrm{mg} / 24 \mathrm{~h})$, and the differences were highly significant $(\mathrm{p}=0.001)$.
Estradiol was at high levels in 5 patients belonging to group I $(380.625 \pm 223.535 \mathrm{pmol} / \mathrm{l})$ and 11 patients from group II (410.531 $\pm 237.838 \mathrm{pmol} / \mathrm{l})$. No statistical significant differences were found $(\mathrm{p}=0.588)$. After performing endovaginal ultrasound to these patients, we found increased ovarian volume with microcysts at the periphery of the ovary. Furthermore, in 4 patients in whom severe nodulocystic and conglobate acne was evident, the endovaginal ultrasound also showed micropolycystic ovaries, but without associated hormonal disturbances.

In group I, no abnormal levels of prolactin and serum cortisol which could impact the development of acne were documented. By contrast, 2 patients $(6.25 \%)$ from group II had hyperprolactinemia $(137.625 \pm 61.901)$, and 4 patients $(12.5 \%)$ had hypercortisolism $(353.969 \pm 102.841)$. The increase in 

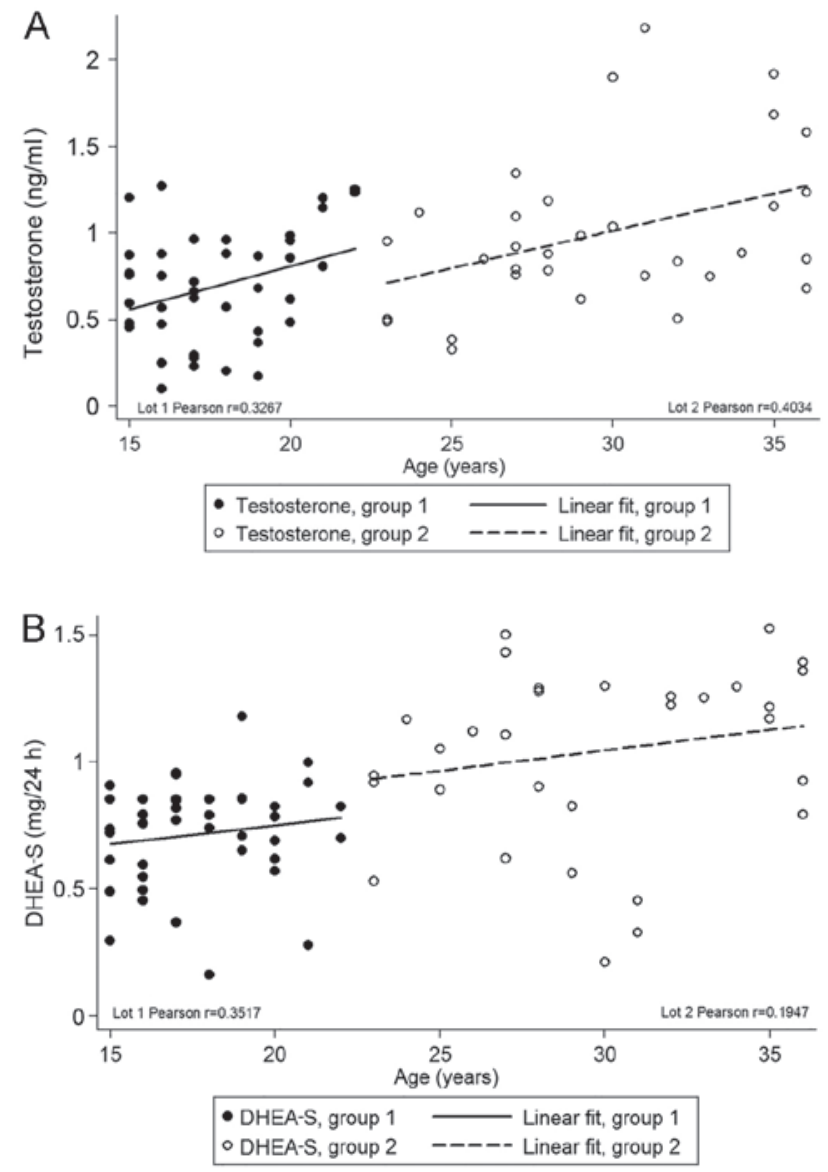

Figure 3. The positive correlation of age with (A) testosterone and (B) DHEA-S levels. DHEA-S, dehydroepiandrosterone-sulfate.

prolactin levels was significant in group II $(\mathrm{p}=0.002)$, while the increase of cortisol was non-significant $(\mathrm{p}=0.182)$.

An LH/FSH ratio $>2$ commonly suggests PCOS and according to this, we registered low levels of FSH in 2 patients (5\%) belonging to group I and LH was increased in 4 patients (10\%) lacking its pulsatile secretion profile. One patient presented PCOS. The hormone levels were $6.31 \pm 3.579 \mathrm{mIU} / \mathrm{ml}$ for FSH and $14.875 \pm 6.711 \mathrm{mIU} / \mathrm{ml}$ for LH. In group II FSH was decreased in 6 patients $(18.75 \%)$ and $\mathrm{LH}$ was increased in 8 patients $(25 \%)$. The FSH levels were $4.545 \pm 3.566 \mathrm{mIU} / \mathrm{ml}$ and the LH levels were $20.156 \pm 8.215 \mathrm{mIU} / \mathrm{ml}$. These hormonal disturbances suggest that in general the hyperandrogeny from endocrine acne progresses in the context of ovarian functional hyperandrogeny. The FSH decrease was statistically significant in patients from group II $(\mathrm{p}=0.041)$ and the LH increase was highly significant in patients from group II $(\mathrm{p}=0.004)$.

After an assessment of age-related hormonal variations, we observed a direct correlation between age and increase in DHEA-S and testosterone levels in patients with refractory acne (Fig. 3).

No significant statistical correlation between abnormal hormonal profiles and the severity of acne was identified.

\section{Discussion}

Acne is a disorder of the pilosebaceous unit that was considered common among adolescents. However, it has been shown that acne may be extended to adulthood, even at the ages of 36-44 years (16). The current treatment of acne vulgaris uses local, laser or intense pulsed light (IPL) therapy (17).

In most of the cases, the poor response to the treatment of acne in teenager females can be related to an unhealthy eating behavior (rich in dairy products or with high glycemic index), an overuse of cosmetic products in order to mask the skin lesions or a decreased compliance to treatment (18).

A high resistance to local of systemic antibiotherapy and a high recurrence rate after use of isotretinoin have been reported (19). These aspects indicate a hormonal etiology of acne and specific treatment should be initiated (20). Hormonal therapy includes: androgen receptor blockers (spironolactone, flutamide, cyproterone acetate) or oral contraceptives, which inhibit ovarian production of androgens and thus reduce sebum secretion (21).

Therapeutic decision should be taken considering the hormonal profile, ultrasound aspect of the ovaries and the localization and severity of acne. In our study, the findings regarding hormonal abnormalities reported in patients from group I cannot be correlated with the presence of refractory acne as only 6 patients presented high testosterone levels and 4 patients had high DHEA-S. The remaining patients did not require hormonal therapy but conventional treatment.

In group II, the hormonal disturbances were much more frequent, as 18 patients were found to have high levels of testosterone and DHEA-S. Patients presented with PCOS, hirsutism, androgenic alopecia and refractory acne and the best option of treatment was represented by blocking agents of the androgenic receptors. We did not find any correlation regarding the hormonal levels and severity of acne. However there was a positive relationship between the clinical manifestation of hyperandrogenism and the clinical form of acne: papulopustular acne was associated with virilisation signs compared with comedonian acne. According to this, 10 of the 16 patients with acne and associated signs of hyperandrogenism had nodular and papulopustular acne, while the other 6 patients had predominantly comedonic lesions.

We also found a positive relationship between the number of acne localizations and the presence of hyperandrogenism signs. To exemplify, at least two locations of acne (most commonly: thorax, shoulders, jaw, neck and cheeks) were associated with irregular menstrual cycles and intense seborrhea of the face and scalp. Thus, the presence of acne on those sites, accompanied by intense facial seborrhea, hirsutism and irregular menses could reveal endocrine disorders that should be thoroughly investigated $(22,23)$.

In our study, the most common localizations of acne were: neck and jaw, 18 patients; perioral, 14 patients; lateral region of the cheeks, 12 patients; anterior and posterior thorax, 8 patients; while 28 patients $(87.5 \%)$ presented at least two regions affected by acne. On the other hand, the absence of the clinical manifestation of hyperandrogenism cannot exclude the coexistence of an endocrine disorder thus, the acne of the adult woman can be considered a hyperandrogenism sign. In our study group, 6 patients without any sign of hyperandrogenism registered high levels of androgens, and 8 patients with obvious clinical signs of hyperandrogenism showed a normal hormonal profile. The results suggest that the increase in androgenic hormones cannot by itself explain refractory acne. 
Patients with adrenal tumors have virilization signs, caused by high testosterone levels, but not always including acne. Many studies sustain the importance of raised testosterone and DHEA-S levels in acne development in women (23). However, in most of the cases, there are not any hormonal dysregulations, suggesting a peripheral malfunction, in the pilosebaceous unit, rather than in the hormone-producing glands. It has been reported that the areas of skin affected by acne are capable of converting testosterone to dihydrotestosterone up to 20 -fold more than unaffected skin, commonly the result of idiopathic functional peripheral hyperandrogenism at the sebocyte level. There are some differences in androgen metabolism by sebaceous glands depending on the body region, thus, facial skin has greater hormonal conversion ability than posterior thorax, which explains the distribution of acne in this region. Thus, sebum production is a sensitive marker of androgenic activity (23).

The present study has several limitations related to the methodology used, including a cross-sectional observational approach, lack of data under therapy and response to androgen receptor blockage or oral contraceptives, as well as heterogeneity of etiology of acne in the study groups.

In conclusion, refractory acne in post-adolescent women is a therapeutic challenge and requires a multidisciplinary approach. Acne can be the sign of PCOS, but the diagnosis needs to be certified through corroboration of both clinical signs and laboratory results. Isotretinoin is an important therapeutic option, albeit hormonal treatment such as androgen receptor blockers and/or oral contraceptives can be recommended even when serum androgen levels are normal, when conventional dermatological treatment alone does not lead to any improvement.

\section{Acknowledgements}

This study received financial support through the project entitled 'Innovative algorithms for diagnosis and treatment of acne' from the operational program POS CCE 2007-2013, grant no. 456/3.04.2013, ID no. 1433/SMIS 41582.

\section{References}

1. Brănişteanu DE, Cotrutz CE, Luca MC, Molodoi DA, Stoica LE, Ianoşi SL, Cianga CM and Brănişteanu DC: Morphopathological stigmata in acne fulminans. Rom J Morphol Embryol 56: 1185-1190, 2015

2. Kamangar F and Shinkai K: Acne in the adult female patient: A practical approach. Int J Dermatol 51: 1162-1174, 2012.

3. Danby FW: New, relevant information and innovative interventions in the management of acne. G Ital Dermatol Venereol 146: 197-210, 2011.
4. Adityan B, Kumari R and Thappa DM: Scoring systems in acne vulgaris. Indian J Dermatol Venereol Leprol 75: 323-326, 2009.

5. Surcel M and Stamatian F: Folliculogenesis disturbances within the polycystic ovarian syndrome and possible consequences on oocyte quality. Acta Endo (Buc) 8: 267-287, 2012.

6. Georgescu CE: Polycystic ovary syndrome endocrine and cardio-metabolic abnormalities: How to manage? Acta Endo (Buc) 11: 77-84, 2015.

7. Köşüş N, Köşüş A and Turhan NÖ: Relationship of ovarian volume with mean platelet volume and lipid profile in patients with polycystic ovary syndrome. Exp Ther Med 2: 1141-1144, 2011.

8. Xia Y, Wang W, Wang L, Shen S, Cao Y, Yi L, Gao Q and Wang Y: hOGGl gene polymorphisms and susceptibility to polycystic ovary syndrome. Biomed Rep 4: 421-426, 2016.

9. Ozdemir S, Ozdemir M, Görkemli H, Kiyici A and Bodur S: Specific dermatologic features of the polycystic ovary syndrome and its association with biochemical markers of the metabolic syndrome and hyperandrogenism. Acta Obstet Gynecol Scand 89: 199-204, 2010.

10. Gudovan E, Diaconescu C, Oros S and Neamtu C: Autoimmune thyroiditis associated with polycystic ovary syndrome: Comments about 25 cases. Acta Endo (Buc) 4: 173-180, 2008.

11. Baldani DP, Skrgatic L, Cerne JZ, Oguic SK, Gersak BM and Gersak K: Association between serum levels and pentanucleotide polymorphism in the sex hormone binding globulin gene and cardiovascular risk factors in females with polycystic ovary syndrome. Mol Med Rep 11: 3941-3947, 2015.

12. Rich P: Hormonal contraceptives for acne management. Cutis 81 (Suppl): 13-18, 2008.

13. O'Connell K and Westhoff C: Pharmacology of hormonal contraceptives and acne. Cutis 81 (Suppl): 8-12, 2008.

14. Bracero $\mathrm{N}$ and Zacur HA: Polycystic ovary syndrome and hyperprolactinemia. Obstet Gynecol Clin North Am 28: 77-84, 2001.

15. Kovacs P: Hyperprolactinemia and polycystic ovary syndrome. Medscape: Accessed: Apr 10, 2003 (http://www.medscape. $\mathrm{com} /$ viewarticle/451707).

16. Yanovski JA, Cutler GB Jr, Chrousos GP and Nieman LK: The dexamethasone-suppressed corticotropin-releasing hormone stimulation test differentiates mild Cushing's disease from normal physiology. J Clin Endocrinol Metab 83: 348-352, 1998.

17. Ianosi S, Neagoe D, Calbureanu M and Ianosi G: Investigator-blind, placebo-controlled, randomized comparative study on combined vacuum and intense pulsed light versus intense pulsed light devices in both comedonal and papulopustular acne. J Cosmet Laser Ther 15: 248-254, 2013.

18. Collier CN, Harper JC, Cafardi JA, Cantrell WC, Wang W, Foster KW and Elewski BE: The prevalence of acne in adults 20 years and older. J Am Acad Dermatol 58: 56-59, 2008.

19. Reynolds RC, Lee S, Choi JY, Atkinson FS, Stockmann KS, Petocz P and Brand-Miller JC: Effect of the glycemic index of carbohydrates on acne vulgaris. Nutrients 2: 1060-1072, 2010.

20. Zhu JT, Xuan M, Zhang YN, Liu HW, Cai JH, Wu YH, Xiang XF, Shan GQ and Cheng B: The efficacy of autologous platelet-rich plasma combined with erbium fractional laser therapy for facial acne scars or acne. Mol Med Rep 8: 233-237, 2013.

21. Lowenstein EJ: Diagnosis and management of the dermatologic manifestations of the polycystic ovary syndrome. Dermatol Ther (Heidelb) 19: 210-223, 2006.

22. Thiboutot D: Acne: Hormonal concepts and therapy. Clin Dermatol 22: 419-428, 2004.

23. Jensterle M, Goricar K and Janez A: Metformin as an initial adjunct to low-dose liraglutide enhances the weight-decreasing potential of liraglutide in obese polycystic ovary syndrome: Randomized control study. Exp Ther Med 11: 1194-1200, 2016. 